\title{
Multiplexed optical data storage and vectorial ray tracing
}

\author{
C. A. Macías Romero, A. S. Van de Nes, M. R. Foreman, P. Munro and P. Török \\ The Blackett Laboratory, Imperial College London, SW7 2BW, United Kingdom
}

\begin{abstract}
With the motivation of creating a terabyte-sized optical disk, a novel imaging technique is implemented. This technique merges two existing technologies: confocal microscopy and Mueller matrix imaging. Mueller matrix images from a high numerical space are obtained. The acquisition of these images makes the exploration of polarisation properties in a sample possible. The particular case of optical data storage is used as an example in this presentation. Since we encode information into asymmetric datapits (see Figure 1), the study of the polarisation of the scattered light can then be used to recover the orientation of the pit. It is thus possible to multiplex information by changing the angle of the mark. The storage capacity in the system is hence limited by the number of distinct angles that the optical system can resolve. This presentation thus answers the question; what is the current storage capacity of a polarisation sensitive optical disk? After a brief introduction to polarisation, the decoding method and experimental results are presented so as to provide an answer to this question. With the aim of understanding high NA focusing, an introduction to vectorial ray tracing is then given.
\end{abstract}

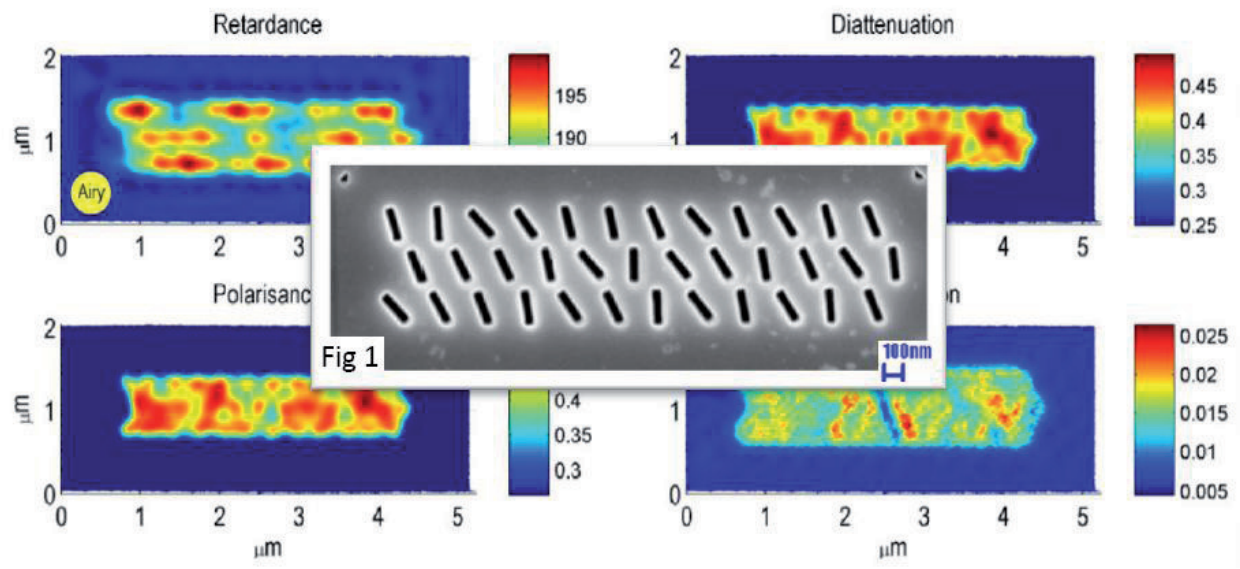

Fig. 1. SEM image of the asymmetric datapits (center). Images of retardance, diattenuation, polarisance and depolarisation obtained with our confocal Mueller matrix microscope.

\section{References}

1. P.R.T. Munro, C. A. Macias-Romero and P Török, Focus on Microscopy Conference, Valencia (2007)

2. D. Lara and C. Dainty, Appl. Opt., 45, 1917 (2006)

3. P. Török, P. Higdon, and T. Wilson, Opt. Commun., 148, 300 (1998)

4. S. -Y. Lu and R. A. Chipman, J. Opt. Soc. Am. A, 13 (1996) 